\title{
DEM simulation of dendritic grain random packing: application to metal alloy solidification
}

\author{
Antonio Olmedilla ${ }^{1, \star}$, Miha Založnik ${ }^{1, \star \star}$, and Hervé Combeau ${ }^{1, \star \star \star}$ \\ ${ }^{1}$ Institut Jean Lamour, CNRS - Université de Lorraine, F-54011 Nancy CEDEX, France
}

\begin{abstract}
The random packing of equiaxed dendritic grains in metal-alloy solidification is numerically simulated and validated via an experimental model. This phenomenon is characterized by a driving force which is induced by the solid-liquid density difference. Thereby, the solid dendritic grains, nucleated in the melt, sediment and pack with a relatively low inertia-to-dissipation ratio, which is the so-called Stokes number. The characteristics of the particle packed porous structure such as solid packing fraction affect the final solidified product. A multi-sphere clumping Discrete Element Method (DEM) approach is employed to predict the solid packing fraction as function of the grain geometry under the solidification conditions. Five different monodisperse noncohesive frictionless particle collections are numerically packed by means of a vertical acceleration: a) three dendritic morphologies; b) spheres and c) one ellipsoidal geometry. In order to validate our numerical results with solidification conditions, the sedimentation and packing of two monodisperse collections (spherical and dendritic) is experimentally carried out in a viscous quiescent medium. The hydrodynamic similarity is respected between the actual phenomenon and the experimental model, that is a low Stokes number, $o\left(10^{-3}\right)$. In this way, the experimental average solid packing fraction is employed to validate the numerical model. Eventually, the average packing fraction is found to highly depend on the equiaxed dendritic grain sphericity, with looser packings for lower sphericity.
\end{abstract}

\section{Introduction}

During metal alloy solidification solid and liquid phases co-exist since solid grains are nucleated in the melt. The solid phase density is slightly higher than that of the melt so the solid particles tend to settle and pack, e.g. aluminum and steel alloys. This forms a porous dendritic network [1] whose properties such as its solid packing fraction are important to better understand in order to improve the solidification macrosegregation modeling of metallic alloys $[2,3]$.

In this article, we focus on the modeling of the random packing phenomenon of the equiaxed dendritic solid grains during solidification and essentially the study of the geometrical influence though sphericity on the packing fraction. The packing is characterized by particles whose morphology is equiaxed dendrite-like and by a low driving force which is the apparent weight of the solid grains immersed in the melt [4].

For the modeling strategy the DEM is chosen, integrating the solid grain dynamics in sedimentation and packing. Plenty of work has already been carried out on this numerical technique for the case of spherical particles, however, much less work can be found for the case of ar-

\footnotetext{
$\star$ e-mail: antonio.olmedilla.aero@gmail.com

$\star \star$ e-mail: miha.zaloznik@univ-lorraine.fr

$\star \star \star$ e-mail: herve.combeau@univ-lorraine.fr
}

bitrary geometry, specially nonconvex particles. Among the different numerical strategies suggested in literature to build a non-spherical morphology, some of them are the spherical-ellipsoidal combination [5], multi-sphere clumping [6] or convex-geometry combinations [7]. In the first strategy the contact detection is based on the contact between two ellipsoids, the second is the simplest contact detection since it is based on the sphere-sphere contact, meanwhile the third strategy is based on the Gilbert-Johnson-Keerthi algorithm to compute the distance between two convex geometries. The second strategy offers the best possibility for the description of arbitrary complex particles. Nevertheless, the computational time is its major weakness.

\section{Model description}

Our algorithm is designed to be an arbitrary-geometry DEM. We model the equiaxed dendritic grain deposition and packing under solidification conditions. In this case the geometry is characterized by having six principal branches grown along the $\langle 100\rangle$ crystal directions. Subsequently, a secondary set of arms are grown from the principal ones [4]. To simplify an envelope wrapping the dendritic branches is considered (see the dendritic envelopes of the experimental model in figure 1a). Eventually the 
envelope surface is precisely discretized by a multi-sphere clumping approach (see figure $1 \mathrm{~b}$ ).

\subsection{Rigid Body Dynamics}

The dynamics equations (eqs. from 1 to 4 ) are solved via the Verlet scheme for each $i$-th grain, obtaining position, $\mathbf{r}$; orientation quaternion, $q$; linear and angular velocities, $\mathbf{v}$ and $\omega$, respectively. Indexes $i r$ and $b$ correspond to inertial reference and body, respectively. $m$ and $\mathbf{I}_{G, b}^{-1}$ are referred to the particle mass and inertial tensor about the particle center of gravity, respectively. Finally, $\mathbf{F}_{t o t}$ and $\mathbf{M}_{G, t o t}$ are the total force and moment applied to the particle center of gravity, respectively.

$$
\begin{gathered}
\frac{d \mathbf{r}_{i r}}{d t}=\mathbf{v}_{i r} \\
\frac{d \mathbf{v}_{i r}}{d t}=m^{-1} \mathbf{F}_{t o t, i r} \\
\frac{d q}{d t}=\frac{1}{2} q \omega_{b} \\
\frac{d \omega_{b}}{d t}=\mathbf{I}_{G, b}^{-1}\left(\mathbf{M}_{G, t o t, b}-\omega_{b} \times\left(\mathbf{I}_{G, b} \omega_{b}\right)\right)
\end{gathered}
$$

\subsection{Geometry discretization}

Spherical elements are set at the particle complex surface in a way that these spheres are internally tangential to the particle surface. This strategy, to face up the particleparticle contact detection, is selected due to their geometrical simplicity and high capacity to describe complex particle shapes.

The dendritic grain morphology is simplified by a geometrical model which consists of 6 quadrilateral pyramids with a central cube. Each of the pyramids is joined to the central cube by its base. We characterize this geometrical configuration by its sphericity, $\Psi$, which is function of the pyramid apex angle, $\theta$, (see eq. 5). Moreover, the characteristic particle size, $l_{c}$, is the distance from a branch tip to opposite branch tip passing along the particle center.

$$
\Psi(\theta)=\sin \left(\frac{\theta}{2}\right)\left\{\frac{\pi}{6}\left[1+\operatorname{cotan}\left(\frac{\theta}{2}\right)\right]^{2}\right\}^{1 / 3}
$$

In figure $1 \mathrm{~b}$ the spherical element approach of complex particle shape description is shown. The spherical elements are uniform and characterized by a diameter $d_{s p h}$. In this specific case, the quadrilateral pyramid apex angle is $45^{\circ}$ and $l_{c} / d_{s p h} \approx 20$.

\subsection{Contact model}

The classical Kelvin-Voigt model is used for the normal reaction in case of contact between the $i$-th and $j$-th particles. Thereby, the contribution to the normal reaction of two contacting spherical elements $l$ and $m$ which belong to the $i$-th and $j$-th particles, respectively, is given by eq. 6 ,
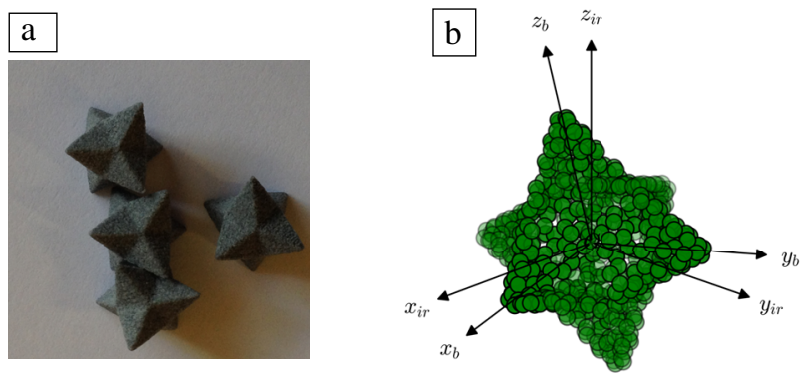

Figure 1. a) Experimental model equiaxed dendritic grain envelope $\left(\theta=45^{\circ}, d_{e q}=7 \mathrm{~mm}\right)$ and $\left.\mathrm{b}\right)$ numerical equiaxed dendritic grain envelope $\left(\theta=45^{\circ}, d_{e q}=7 \mathrm{~mm}\right)$. The surface is described with 527 spherical elements $\left(l_{c} / d_{s p h} \approx 20\right)$. The indexes ir and $b$ refer to inertial reference and body frames, respectively.

where the stiffness and viscous damping dissipation reactions are summed. In the general case of non-uniform particle contact mechanical properties, the stiffness, $K_{l m}$, and damping, $C_{l m}$, coefficients are referred to the $l-m$ pair of contacting spherical elements. The variables $\delta_{l m}$ and $\dot{\delta}_{l m}$ refer to the overlapping and overlapping rate between the pair of spherical elements $l-m$. A critical damping coefficient is used in order to avoid rebounding, i.e. dissipating the overall settling particle kinetic energy at the contact.

$$
F_{i l, j m}^{n}=K_{l m} \delta_{l m}+C_{l m} \dot{\delta}_{l m}
$$

\section{Simulation cases}

\subsection{Domain and initial conditions}

A rectangular prismatic domain is employed whose dimensions are $L_{x}, L_{y}$ and $L_{z}$, with the direction $z$ considered as vertical and $x-y$ directions forming a horizontal plane. In the simulations presented in this article, the horizontal cross-section of the prism is square, $L_{y}=L_{x}$ and the vertical dimension is larger than the horizontal $L_{z}>L_{x}$. Additionally, the domain walls are rigid in order to obtain the wall effect influence on the packing.

Initially particles are randomly distributed withing the whole domain occupying a low solid fraction $(\approx 4 \%)$ without initial contacts. In this way, their positions are initialized by a Random Sequential Addition algorithm [8]. Meanwhile the particle initial orientations are randomly computed by the K. Shoemaker algorithm [9].

Dynamic initial conditions of linear and angular velocities are null.

\subsection{Packing protocol}

The overall granular system simultaneously sediments and packs by means of a vertical uniform relatively low acceleration $\left(a=10^{-5} g_{o}\right)$ over the horizontal domain wall $z=0$. The objective of this protocol is that of simulating the equiaxed dendritic grain sedimentation and packing with an equivalent inertia-to-dissipation ratio to that in solidification and in the validation experimental model but 


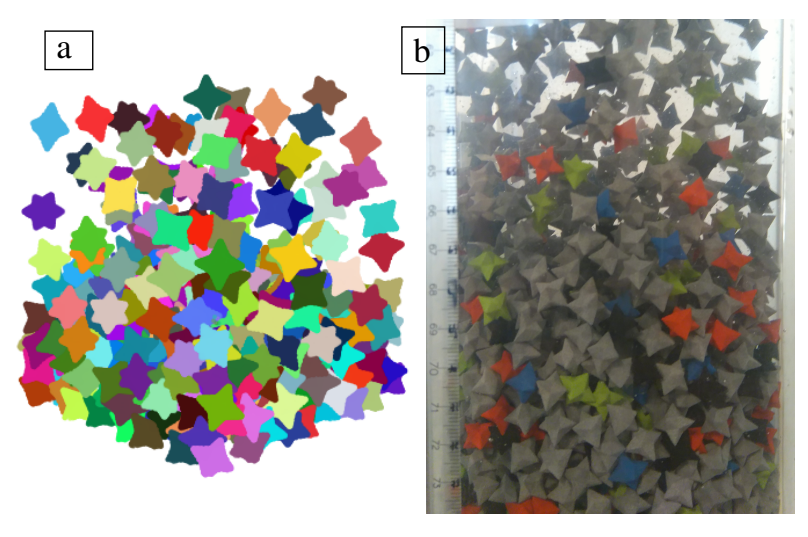

Figure 2. Random packing of equiaxed dendritic grain envelope $\left(\theta=45^{\circ}, d_{e q}=7 \mathrm{~mm}\right)$ : a) DEM simulation, b) Experimental validation packing.

decreasing the mathematical stiffness by means of a low driving force and consequently, increasing the integration time step. This protocol considerably accelerates the simulation permitting to achieve higher particle geometry detail.

In the actual solidification phenomenon and in the validation experimental model, the granular system kinetic energy is dissipated through the lubrication forces at the tight fluid layers among the grains. In this numerical model, the kinetic energy dissipation is activated at the grain contact through the critical viscous damping term in eq. 6 . The critical contact stiffness is selected in order to reproduce the equivalent inertia-to-dissipation ratio once the driving force is chosen.

\subsection{Simulated geometries}

- a) Dendrite-like: three different 1000 monodisperse dendrite noncohesive frictionless collections are packed in a $84 \times 84 \times 500 \mathrm{~mm}^{3}$ domain. The three of them has an equivalent diameter of $d_{e q}=7 \mathrm{~mm}$. Three different pyramid apex angles are simulated.

- Dendrites of $45^{\circ}$ apex angle and $\Psi=0.69$ (figure $1 \mathrm{~b}$ ).

- Dendrites of $60^{\circ}$ apex angle and $\Psi=0.79$.

- Dendrites of $90^{\circ}$ apex angle and $\Psi=0.90$.

- b) Spherical: 2000 monodisperse noncohesive frictionless particle collection whose diameter is $7 \mathrm{~mm}$ in a $84 \times 84 \times 1000 \mathrm{~mm}^{3}$ domain.

- c) Ellipsoids of $1.25: 0.8: 0.8$ axis ratio and $\Psi=0.97$.

The particle density is uniform and the same for all particle collections $\left(\rho_{s}=1400 \mathrm{~kg} / \mathrm{m}^{3}\right)$. The particle density and equivalent diameter between the simulation and the validation experiments are identical.

\subsection{Numerical results}

Once the granular system achieves the mechanically stable equilibrium, the kinetic system energy is dissipated by means of the viscous damping contact, the average packing fraction at $\Omega$ volume, $\left\langle\phi_{s}\right\rangle_{\Omega}$, is obtained by computing the Voronoi tessellation of the granular system by means of the Voro++ open library [10] and subsequently computing the average value of a certain volumetric region, $\Omega$, applying eq. 7 , where $V_{s_{i}}$ and $V_{\text {cell }_{i}}$ are the volume of the $i$-th particle and its corresponding Voronoi cell volume, respectively. The region $\Omega_{A}$ is defined by the intervals $[0,84] \times[0,84] \times[0, h] \mathrm{mm}^{3}$ where $h$ is the lowest position of the top packing surface whereas $\Omega_{B}$ is defined by $[22,62] \times[22,62] \times[10,20] \mathrm{mm}^{3}$.

$$
\left\langle\phi_{s}\right\rangle_{\Omega}=\frac{\sum V_{s_{i}}}{\sum V_{\text {cell }_{i}}}, \forall i / \mathbf{r}_{i} \in \Omega
$$

In the case of spheres $(\Psi=1)$, we obtain a value of $\left\langle\phi_{s}\right\rangle_{\Omega_{B}}=0.643$ which corresponds to the result in [11] for sedimentation and pack with relatively low driving force. A slightly looser packing fraction is obtained when the wall effect is accounted $\left\langle\phi_{s}\right\rangle_{\Omega_{B}}=0.618$ (see figure 3 for $\Psi=1$ ). Besides, the ellipsoidal results are also compared to the same literature reference [11], obtaining a $4 \%$ looser packing in our case (see figure 3 for $\Psi=0.97$ ).

The three different dendritic geometries are sedimented and packed (see figure 2a) and the same Voronoi tessellation procedure is employed to obtain the local volumetric solid packing fraction for later computing the average packing fraction at a desired region $\left(\Omega_{A}\right.$ and $\left.\Omega_{B}\right)$.

A clear influence of the equiaxed dendritic grain sphericity on the packing fraction is found: the lower the grain sphericity, the looser the grain packing is. The equiaxed dendritic envelopes whose $\Psi=0.69$ pack an approximately $38 \%$ looser than spheres (fig. 3 ).

The vessel wall effect (the difference $\left\langle\phi_{s}\right\rangle_{\Omega_{B}}-\left\langle\phi_{s}\right\rangle_{\Omega_{A}}$ ) is shown to slightly increase with decreasing sphericity for equiaxed particles (spheres and dendrites).

A numerical simulation correlation of sequential deposition of spiky nonconvex particles [5] are included in fig. 3 since the particles in this reference are the most similar found to our dendritic envelopes. For larger sphericity $\Psi>0.85$ our results and this reference results are similar. However, a great behavior difference is found for lower sphericity $\Psi=0.70$, being the dendritic envelopes denser packed.

Additionally, our numerical packing results of equiaxed dendritic envelopes under low inertia-todissipation conditions are validated by means of an experimental model (see sec. 3.5). The numerical and experimental results are compared through $\left\langle\phi_{s}\right\rangle_{\Omega_{A}}$ since the experimental results include the vessel wall effect. In the case of spheres, our numerical protocol obtains denser packings $\left(7.3 \%\right.$ difference) whereas in case of the $45^{\circ}$ equiaxed dendritic envelopes a slightly lower numerical fraction is found $(6.7 \%)$. In this way, arbitrary-geometry DEM and packing protocol is validated to reproduce equiaxed dendritic envelope packings under solidification conditions (low inertia-to-dissipation). 


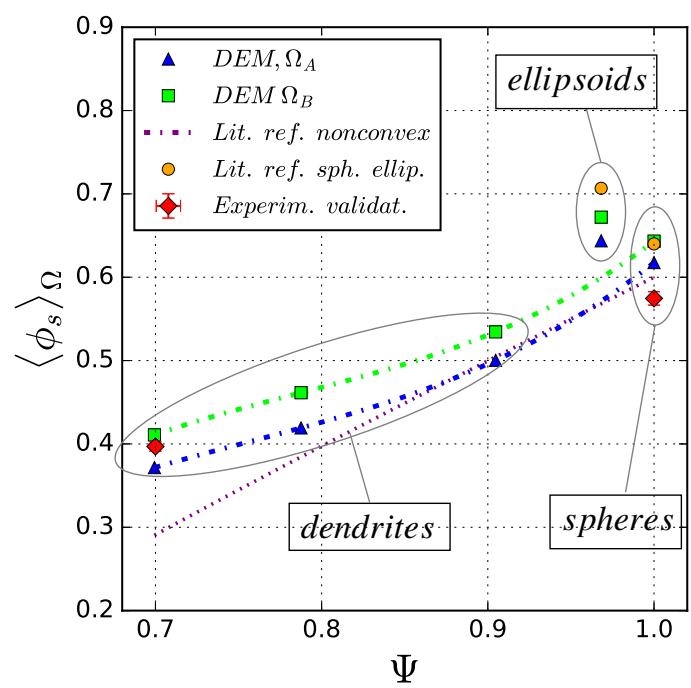

Figure 3. (Color online) Average solid volumetric packing fraction, $\left\langle\phi_{s}\right\rangle_{\Omega}$, function of particle sphericity. $\left\langle\phi_{s}\right\rangle_{\Omega_{A}}$ (accounting the vessel wall effect) is shown with triangular blue markers) and $\left\langle\phi_{s}\right\rangle_{\Omega_{B}}$ shown via squared green markers). The equiaxed grain results are joined by a dashed green and blue lines, $\left\langle\phi_{s}\right\rangle_{\Omega_{B}}$ and $\left\langle\phi_{s}\right\rangle_{\Omega_{A}}$, respectively. The validation experiments (see sec. 3.5) for $45^{\circ}$ dendritic envelopes and spheres are shown via red rhomboid markers. The numerical results from literature for spheres and ellipsoids under low gravity conditions [11] (Lit. ref. ellip.) and sequential deposition under gravity of spiky nonconvex particles [5] (Lit. ref. non-convex) are also included.

\subsection{Validation experimental model}

In order to validate the DEM solver and packing protocol, a 12200 cornstarch sphere and a 44003 d-printed $45^{\circ}$ apex-angle dendrite monodisperse collections are packed by sedimentation in a cylindrical vertical sedimentation column containing a $99.5 \%$ glycerol solution with a vessel diameter to particle equivalent diameter ratio is $D / d_{e q}=$ 17 (see fig. 2b). The density of both particle collections is approximately $1400 \mathrm{~kg} / \mathrm{m}^{3}$. The liquid is chosen in order to obtain a Stokes dimensionless number $\left(S t=\left(\rho_{s} d_{e q} v_{s}\right) /\left(9 \mu_{l}\right)\right)$ of $10^{-3}$ which is a characteristic value in solidification. The average solid packing fraction is obtained for both sphere and dendrite collections by carefully measuring the height of the packed system with a 10 repetitions for each geometry.

A $0.575 \pm 0.008$ and $0.397 \pm 0.013$ are found for the average solid packing fraction of monodisperse spheres and $45^{\circ}$ dendrites, respectively (see fig. 3 ).

These experimental packings (sedimentation and packing in quiescent viscous fluid) are compared to the literature fluidized packings, which are frequently used to obtain the random loose packing configuration. In this vein, in [12] an average packing fraction of 0.55 is found for monodisperse spheres of diameter of the order of $100 \mu \mathrm{m}$ and a packing fraction in the interval $0.50-0.55$ for tetrahedrons and octahedrons [13].

\section{Conclusions}

The random packing of equiaxed dendritic grains, phenomenon present in metallic-alloy solidification, is numerically studied by a DEM model. Looser packings are obtained with lower particle sphericity under low inertia-todissipation conditions. For the case of $\Psi=0.70$ equiaxed dendritic envelope, an average solid packing fraction of approximately 0.38 is numerically found, which is much lower than that of the monodisperse spheres.

\section{Perspectives}

Future improvements of the arbitrary-geometry DEM model are the implementation of the fluid effects on the particles (drag and lubrication forces), particle polydispersity and particle growth (by scaling).

\section{Acknowledgements}

This work was supported by the French State through the program "Investment in the future" operated by the National Research Agency (ANR) and referenced by ANR11 LABX-0008-01 (LabEx DAMAS).

\section{References}

[1] I. Steinbach, JOM 65, 1096 (2013)

[2] H. Combeau, M. Založnik, S. Hans, P.E. Richy, Metallurgical and materials transactions B 40, 289 (2009)

[3] M. Založnik, A. Kumar, H. Combeau, M. Bedel, P. Jarry, E. Waz, Advanced Engineering Materials 13, 570 (2011)

[4] J.A. Dantzig, M. Rappaz, Solidification, 1st edn. (EPFL Press, 2009)

[5] I. Malinouskaya, V. Mourzenko, J.F. Thovert, P. Adler, Physical Review E 80, 011304 (2009)

[6] Christophe L. Martin, Mechanics of Materials with Discrete Element Simulations, http://simap.grenoble-inp.fr/gpm2/ m-christophe-martin--430244.kjsp (2016), online; accessed March 2016

[7] A. Wachs, L. Girolami, G. Vinay, G. Ferrer, Powder Technology 224, 374 (2012)

[8] A. Donev, Ph.D. thesis, Princeton University (2006)

[9] K. Shoemaker, Animating rotation with quaternion curves, in ACM SIGGRAPH computer graphics (ACM, 1985), Vol. 19, pp. 245-254

[10] C. Rycroft, Lawrence Berkeley National Laboratory (2009)

[11] G.W. Delaney, J.E. Hilton, P.W. Cleary, Phys. Rev. E 83, 051305 (2011)

[12] M. Jerkins, M. Schröter, H.L. Swinney, T.J. Senden, M. Saadatfar, T. Aste, Phys. Rev. Lett. 101, 018301 (2008)

[13] J. Baker, A. Kudrolli, Physical Review E 82, 061304 (2010) 